\title{
CANCER OF THE PENIS
}

\author{
MD. WALIUL ISLAM ${ }^{1}$, MD. ABUL HOSSAIN ${ }^{2}$, MD. NURUL HOODA ${ }^{1}$, MD. AM ANAMUR RASHID \\ CHOUDHURY ${ }^{1}$, MD. MAMUNUR RASHID ${ }^{1}$, SARFORAJ ALI KHAN ${ }^{1}$, MD. SHAHIDUL ISLAM ${ }^{3}$, M.A. AWAL ${ }^{4}$ \\ ${ }^{1}$ Dept. of Urology, National Institute of Kidney Diseases \& Urology, Dhaka. ${ }^{2}$ Dept. of Urology, Shaheed Suhrawardy Medical \\ College, Dhaka. ${ }^{3}$ Dept. of Urology, Rangpur Medical College, 4. Dept. of Urology, Dhaka Medical College, Dhaka.
}

\begin{abstract}
Objectives: To review the outcomes of modification of groin lymph nodes dissection on morbidity from surgical treatment of a patient with penile cancer and to find out accurate treatment plan according to stages of the primary lesion from current literatures.

Methods: We searched the pubmed database for English Language published in the past for years using incidence, etiology, pathology, clinical presentation, staging and management of penile cancer.

Result: From retrieved literatures better understanding of pathologic features allow for stratification of patients into low, intermediate, or high risk for lymph node involvement. Lymphatic mapping to this stratification improves selection of patients who might benefit from lymph node dissection after excision of the primary lesion. The recent use of a modified lymph node dissection has minimized morbidity. Current chemotherapy agents are ineffective in this disease.

Conclusion: Patholoigc features of the primary lesion and the incorporation of lymphatic mapping have improved the selection of patients who might benefit from lymph node dissection. The use of a modified lymph node dissection in selection patients has decreased morbidity. Effective chemotherapy agents are needed in the management of advanced penile cancer.
\end{abstract}

Key words: Penile cancer, Modified inguinal lymphodenopathy staging of penile cancer, lymphodenopathy, pathology of penile cancer.

Bangladesh J. Urol. 2012; 15(2): 48-55

\section{Introduction:}

Penile cancer is an uncommon malignancy but sometimes devastating for the patient. It is typically a disease of middle aged to older men, most commonly affecting those between 50 to 70 years of age. But the cancer is not unusual in younger men. Although rare in the developed countries, penile cancer constitutes a substantial health cancer in many African, South American and Asian Countries.

As it is an uncommon disease so progress in the diagnosis and treatment has occurred slowly. So, there is a scope of new diagnostic and therapeutic techniques as well as attempts to identify patients who may be benefited from less invasive treatment.

Correspondences: Dr. Md. Waliul Islam, National Institute of Kidney Diseases \& Urology, Mobile: 0181925840 , E-mail: maruf.401 @ovi.com
Incidence

The incidence of penile cancer in less than 1 man in $1,00,000$ and accounts for ten than $1 \%$ of cancers in men in the united stages. It accounts for upto $10 \%$ in cancers in some parts of Asis, African and South American.

\section{Etiology}

The most important etiologic factor of penile cancer is the presence of an intact foreskin. Penile cancer is rarely seen in Jewish individuals, who are circumcised at birth In the United State, the risk of this disease in uncircumcised men is 3 -fold higher than that of circumcised men as approaches the rate seen in some underdeveloped nations.

Maden et al reported a study of 110 men with penile cancer and 355 control subjects. The risk of penile cancer was 3.2 times greater among uncircumcised men 
compared with men circumcised at birth and 3.0 times greater among those who had been circumcised after the neonatal period ${ }^{1}$.

Schoen and colleagues evaluated the relationship between newborn circumcision and invasive penile cancer among adult men who were members of a large health maintenance organization ${ }^{2}$. Of 89 men with invasive penile cancer whose circumcision status was known, $2(2.3 \%)$ had been circumcised as newborns and 87 were not circumcised. This study confirms the highly protective effect of newborn circumcision against invasive penile cancer.

A history of phimosis (ie, narrowness of the opening of the prepuce) is found in approximately $25 \%$ of penile cancer patients. Phimosis is strongly associated with invasive carcinoma of the penis. Precancerous lesions are found in an additional $15 \%$ to $20 \%$ of patients.

An ongoing focus of research in the study of penile cancer is the association of human papilloma virus (HPV) with both benign and malignant lesions of penis. Rubin et all evaluated the prevalence of HPV DNA in different histological subtypes of penile carcinoma, dysplasia, and condyloma ${ }^{3}$. HPV DNA was detected in $42 \%$ of cases of penile carcinoma, in $90 \%$ of cases of dysplasia, and in $100 \%$ of cases of condyloma.

Many studies have shown the presence of HPV types 16 and 18 in penile carcinoma. In an examination of 30 specimens of penile cancer by polymeraase chain reaction and in situ hybridization assays from 23 patients, the HPV-16 genome was found in 15 patients (65\%), HPV-30 in 3 patients (13\%), and HPV-6 or HPV11 in 2 patients (9\%). Recently Bezerra et all detected HPV DNA in 25 of 82 samples (30.5\%). HPV-16 was the most frequent type detected (13 of $25,52 \%)^{4}$. Researchers believe that infection with HPV is an important risk factor for penile cancer.

Dillner et all evaluated the etiology of squamous cell carcinoma of the penis ${ }^{5}$. In this study, strong risk factors $(>10)$ identified by case-control studies included phimosis, chronic inflammatory conditions (eg, balanoposthitis and lichen sclerosus et atrophicus), and treatment with psoralen and ultraviolet $A$ photochemotherapy (PUVA). A consistent association was found between penile cancer and smoking that was dose-dependent and not explained by confounding factors such as sexual history. Sexual history and selfreported history of condyloma were associated with a 3-fold increase in penile cancer risk. The authors also cite a cse-control study showing that circumcision neonatally, but not after the neonatal period, was associated with a 3-fold decreased risk of penile cancer. Finally, in a large number of case series, HPV DNA was positive in invasive penile cancer in $40 \%$ to $50 \%$ of cases.

\section{Pathology}

Malignancies of the penis are divided into primary malignancies (ie, those that originate from either the soft tissues, urethral mucosa, or covering epithelium) and secondary malignancies (ie, those that represent metastatic disease and often affect the corpus cavernosum). The first step in the histological diagnosis of malignancy is the confirmation of the diagnosis and assessment of depth of invasion by microscopic examination of a biopsy specimen. A dorsal slit is often necessary to gain adequate exposure to the lesion.

Secondary malignancies (metastatic tumors) should be suspected in patients with a know diagnosis of cancer and who also present with new-onset priapism (involvement of the corpora cavernosum) or an unusual penile lesion. Metastatic lesions are often multiple, palpable, painless nodules that may mimicsyphilitic chancers. The primary malignancy is most often prostate, followed by bladder, rectosigmoid colon, and kidney, and it is spread most commonly by retrograde venous dissemination.

Primary, nonspquamous malignancies comprise less than $5 \%$ of penile cancers. Sarcomas are the most frequent nonsquamous penile cancers, followed by melanomas, basal cell carcinomas, and lymphomas. Kaposi's sarcoma, once isolated only in elderly men, has increased in frequency with the onset of AIDS. The lesion presents as a well-marginated red nodule, often isolated to the glens, and represents the initial site of presentation in $3 \%$ of patients.

Carcinoma in situ (CIS) reflects the full thickness alteration in the epithelium with loss of polarity, multiple atypical mitoses, and increased hyperchromatic cells and multinucleated cells isolated strictly to the epithelium and with an intact basement membrane, Erythroplasia of Queyrat refers to CIS involving the glans penis, prepuce, or shaft, whereas Bowen's disease refers to CIS involving the remainder of the genitalia or perineal region. A third, histologically identical lesion, bowenoid papulosis, has a clinically benign course and often presents in young men (mean age of 29.5) who are sexually active and circumcised. Bowenoid papulosis appears as multiple, pigmented red to brown papular 
lesions that can spontaneously regress in a number of cases yet poses no risk of malignant degeneration.

The majority of squamous cell carcinomas arise de novo. Jensen et al presented a series of 511 patients with penile squamous cell carcinoma ${ }^{6}$. Only $39(7.6 \%)$ had a history of previous or concomitant penile lesions. Squamous cell carcinoma is diagnosed $b$ the histological confirmation on biopsy of invasion through the basement membrane. At presentation, squamous cell carcinoma is found on the glans in $48 \%$ of cases, the prepuce in $21 \%$, glans and prepuce in $9 \%$, coronal sulcus in $6 \%$, and shaft in $<2 \%$. The histopathologic grading is based on the Broder's Classification System. In this system, grade I consists of cells well differentiated with keratinization, prominent intercellular bridges, and keratin pearls. Grade II to III includes greater nuclear atypia, increased mitotic activity, and decreased keratin pearls. Grade IV cells are deeply invasive and consist of marked nuclear pleomorphism, nuclear mitoses, necrosis, lymphatic and perineural invasion, and no keratin pearls.

Half of lesions presenting on the penile shaft are poorly differentiated, whereas only $10 \%$ of lesions on the prepuce are well differentiated. Cubilla and group associated the morphology of the primary lesion to disease progression ${ }^{7}$. Superficially spreading squamous cell cancers occur most frequently and present with lymph nodes positive for metastatic disease in $42 \%$ of cases. Lesions with a deeper vertical growth present with positive lymph nodes in $82 \%$ of cases. Multicentric lesions have positive nodes in $33 \%$, whereas verrucous lesions rarely present with metastasis to lymph nodes. Differences between exophytic and ulcerative lesions have also been described. Patients with ulceration have a higher likelihood of node-positive disease.

\section{Clinical Presentation}

The initial evaluation of any suspected penile lesion relies on clinical examination of both the primary tumor and inguinal lymph nodes. The clinical presentation of an ivasive penile carcinoma is varied and may range from an area of induration or erythema to a nonhealing ulcer or a warty exophytic growth. Phimosis may obscure the tumor, thus possibly delaying diagnosis of the tumor until a bloody or foul-smelling discharge occurs.

All penile lesions, particularly those under a nonretractile foreskin, require a high index of suspicion for neoplasia. A penile lesion that does not resolve after 2 to 3 weeks of carefull observation and skin care requires biopsy. If possible, biopsies should include enough tissue to determine the depth of invasion since this will ultimately affect therapy. In large lesions, the diagnosis is obtained by incisional biopsy.

In decreasing order of frequency, penile cancer develops in the glans $(48 \%)$, prepuce $(21 \%)$, glans and prepuce $(9 \%)$, coronal sulcus $(6 \%)$, and shaft $(<2 \%)$. Careful palpation of the inguinal regions is important since palpable inguinal lymphadenopathy is present at diagnosis in $58 \%$ of patients (range $20 \%$ to $96 \%$ ), and metastatic carcinoma will ultimately be diagnosed in $45 \%$ of these patients. The remainder will have inflammatory lymphadenopathy that resolves following resection of the primary tumor and a 4- to 6-week course of oral antibiotics. In patients with nonpalpable inguinal lymph nodes at the time of resection of the primary tumor, $20 \%$ will ultimately be found to have metastatic disease in the superficial groin nods. Late in the course of the disease, metastasis to retroperitoneal nodes, liver, lung, and brain can occur. Bulky unresectable nodes can erode into the femoral vessels leading to exsanguinating hemorrhage.

The physical examination is key to the clinical evaluation of the patient with penile cancer. The assessment of the primary tumor should include the size, location, fixation, and involvement of the corporal bodies. The penile base and scrotum should be inspected to exclude neoplastic extension. Both groins should be palpated for inguinal lymphadenopathy.

\section{Staging}

A staging system is a standardized way to summarize information about how far a cancer has spread. Two staging systems are used in penile carcinoma: The Jackson classification (Table-I) ${ }^{8}$ and the TNM classification (Table-II) ${ }^{9}$. From a historical point of view, Jackson's staging system represent's the original penile cancer staging system. In the current TNM system the primary tumor is staged according to histological features and the precise anatomic structure invaded.

Table-I

Jackson's classification for Ca-penis

\begin{tabular}{|c|c|}
\hline Stage-I & : Tumor confirmed to glans, prepares \\
\hline Stage-II & : Tumor extending on to shaft of penis. \\
\hline Stage-III & $\begin{array}{l}\text { : Tumor with inguinal metastases that are } \\
\text { operable. }\end{array}$ \\
\hline Stage-IV & $\begin{array}{l}\text { Tumor involving adjacent structures tumor } \\
\text { associated with inoperable inguinal } \\
\text { metastasis or distant metasis. }\end{array}$ \\
\hline
\end{tabular}


Table-II

TNM classification of tumors of the penis.

\begin{tabular}{ll}
\hline Tx: & Primary tumor \\
To: & Cannot be assessed \\
Tis: & No evidence of primary tumor \\
Ta: & Noninvasive verrucous carcinoma \\
T1: & Invades subepithelial connective tissue \\
T2: & Invades corpus spongiosum or cavernosum \\
T3: & Invades urethra or prostate \\
T4: & Invades other adjacent structures. \\
N- & Regional lymph nodes. \\
Nx: & Cannot be assessed \\
No: & No regional lymph node metastasis \\
N1: & Metastasis in single superficial inguinal node \\
N2: & Metastasis in multiple or bilateral superficial inguinal \\
& nodes. \\
N3: & Metastasis in deep inguinal or pelvic node \\
M- & Distant metastasis \\
Mx: & Cannot be assessed \\
Mo: & No distant metastasis \\
M1: & Distant metastasis is present. \\
\hline
\end{tabular}

Leijte et al have proposed some changes for better prognostic differentiation, but these changes have yet to be widely implemented ${ }^{10}$.

\section{Prognostic Factors}

The grade of the primary tumor and depth of invasion are considered to be the most important indicators for metastatic spread. Recently, tumor of vertical growth and with vascular or lymphatic invasion are considered prognostic indicators for metastatic spread ${ }^{11,12}$.

\section{Surgical Treatment}

The aim of treatment of penile cancer is to optimize loco-regional and systemic disease eradication with preservation of penile function and minimal disfiguative.

\section{Treatment of the Primary Lesion:}

Small tumors limited to the foreskin can be treated by circumcision with a $2-\mathrm{cm}$ margin of clearance. Circumcision alone, especially with tumors in the proximal foreskin, may be associated with recurrence rates of $32 \%$. These high recurrence rates under score the need for careful follow-up of patients treated by circumcision alone. Local recurrence, as high as $56 \%$, most commonly occurs after organ-preserving procedures. A pathological assessment of surgical margins is essential in apply these procedure and to reduce the rate of local recurrence ${ }^{13}$.
Mohs micrographic surgery is used for the treatment of selected patients with small, superficial penile cancers $^{14}$. The Mohs technique involves ecision of the penile lesion and microscopic examination of the underside of each layer for systematic inspection of serial sections. Two techniques are used for the complete microscopic control of the excision of the cancer: (1) the fixed-tissue technique, in which the tissues are fixed with zinc chloride paste before the excision of the successive layer and (2) the fresh-tissue technique, in which the tissues are excised in the fresh, unfixed state and evaluated by frozen section. A study by Mohs and colleagues reoirted ib 35 patients with squamous cell carcinomas of the penis who were treated by means of micrographic surgery. Eight patients recurred, with 6 developing uncontrolled metastases and 2 with tumor extension along the urethra. The 5 year cure rate and the overall local 5year cure rate was $74 \%$, and $94 \%$, respectively.

The use of laser as on organ sparing therapy for penile carcinoma was first reported by Hofstetter et al in $1978^{15}$. Laser ablation using a neodymium: ytrium-aluminum garnet (Nd:YAG) or carbon dioxide $\left(\mathrm{CO}_{2}\right)$ laser also has been used in selected patients with small superficial or superficially invasive penile cancers.

Radiation therapy is indicated usually for $T_{1}-T_{2}$ tumors that are less than $4 \mathrm{~cm}$. Radiation therapy has yielded local control rates similar to surgical resection. Radiation therapy techniques include external-beam irradiation, iridium molds or wires, and interstitial brachytherapy. Using external-beam radiation therapy, Almgard and Edsmyr achieved a control rate of only $50 \%$. For T1-T2 penile carcinoma, an overall local control rate of $74 \%$ to $86 \%$ was achieved with Ir interstitial implants and $80 \%$ with brachytherapy.

Carcinomas of the penis involving the glans and distal shaft are best managed by partial penectomy margin of the tumor. This should leave a 2.5 to $3 \mathrm{~cm}$ stump of penis to allow directable micturition in a standing posture, with some coital function as well. Recently, evaluated the possibility of reducing the margin of clearance at surgery for carcinoma of the penis without causing an increase in the incidence of local tumor recurrence in order to minimize the functional and cosmetic compromise associated with penectomy. Of 64 patients who underwent partial or total penectomy, 20 patients (31\%) were grade I, $32(50 \%)$ were grade II, and the remaining $12(19 \%)$ were grade III. The maximum histological extent beyound the clinical lesion was 5 
$\mathrm{mm}$ for grades I and II tumors and $10 \mathrm{~mm}$ for grade III tumors. The authors concluded that a $10 \mathrm{~mm}$ clearance is adequate for grade I and II lesions, and $15 \mathrm{~mm}$ clearance for grade III fumors, leaving a more acceptable penile stump length ${ }^{16,17,18}$.

For bulky T3 or T4 proximal tumors involving the base of the penis, total penectomy with perineal urethrotomy is done. These proximal tumors are often advanced and associated with regional metastatic disease.

After 4 to 6 weeks of oral antibiotics following treatment of the primary lesion, the patient is reevaluated for the presence of palpable regional lymphadenopathy. The presence of palpable inguinal lymph nodes at the time of diagnosis may be due to inflammatory reaction or metastatic disease. Only $50 \%$ of patients presenting with palpable lymphadenopathy actually have metastatic disease, the remainder having lymph node enlargement secondary to inflammation. The development of new adenopathy during follow-up is more likely due to tumor in $70 \%$ of cases.

The morbidity of inguinal lymphadenectomy may be considerable, with a $30 \%$ to $50 \%$ incidence of severe lymphedema and skin flap necrosis.

A modification of the standard inguinal lymphadenectomy has been developed by Catalona as another option for patients with clinically negative inguinal lymph nodes $^{19}$. The procedure differs from the standard lymphadenectomy in that the length of the skin incision is reduced, the subcutaneous tissue superficial to Scarpa's fascia is preserved, the dissection is reduced to exclude the regions lateral to the femoral artery and caudal to the fossa ovalis, the saphenous vein is preserved, and transposition of the sartorius muscles is eliminated. In patients with histologically negative inguinal nodes, pelvic lymphadenectomy is not performed. In patients with positive lymph nodes on frozen section, a bilateral iliac lymphadenectomy also is performed.

The technique has been associated with less morbidity than the standard lymphadenectomy, and the results are encouraging. Colberg and colleagues reported on 9 patients who underwent modified inguinal lymphadenectomy ${ }^{20}$. Three had histologically positive lymph nodes, and none of the patients with positive or negative nodes had evidence of recurrent disease. All patients were alive with a mean follow-up of 67.5 months (range 13 to 108 months). Early post-operative complications occurred in 2 patients with skin-flap necrosis, in 1 with prolonged lymphatic drainage, and in 1 with a delayed groin lymphocele and cellulitis. In another study, Parra performed a modified inguinal lymphadenectomy on 12 patients. Five patients were identified with nodal metastasis. The sites of inguinal node involvement were localized within the boundaries of the dissection in all patients. No major complications occurred, and no permanent lymphedema or flap necrosis was encountered. Follow-up of 14 to 72 months demonstrated no disease. However, the reliability of this procedure in detecting histological metastases in patients with clinically negative groin nodes has been questioned.

The timing of lymph node dissection is important, particularly for patients who present with no clinical sign of metastatic disease. Proponent of an early or immediate lymphadenectomy note that patients who do not undergo surgery but are observed often present at a stage where cure is no longer possible. Horenblas and colleagues proposed observation in patients with clinically negative nodes. Patients were followed every 2 months for 3 years, and almost all patients who developed lymph node metastases after treatment of the primary tumor were salvaged by lymph node dissection. Unreliable patients or others who cannot be followed adequately every 2 months are best served by prompt inguinal lymphadenectomy. Solsona et al proposed three risk groups - low, intermediate and high - for occult lymph node metastases in patients with penile carcinoma and clinically negative lymph nodes ${ }^{21}$. Low-risk patients had stage T1/grade I tumors. None of these patients developed lymph node metastasis on follow-up. Intermediate risk group were patients with stage T1/grades II-III tumors or stages T2-3/grade I tumors. These patients had a $36.4 \%$ incidence of positive lymph nodes on follow-up. High risk group were patients with stages T2-3/grade II-III tumors with an $80 \%$ incidence of lymph node involvement on follow-up.

In patients with unilateral nodal recurrence during the follow-up, bilateral lymph node dissection should be done. In these cases, the probability of occult contralateral involvement is $60 \%$ to $79 \%$ of cases due to crossover lymphatics at the base of the penis.

Metastasis to the pelvic nodes in the absence of inguinal node metastasis is a rare event and has not been observed in many modern series. In the setting of negative superficial and deep inginal lymphadencetomies and a negative pelvic computed tomography scan, pelvic lymphadenectomy is not required. 


\section{Lymphoscintigraphy and Sectinel Node Biopsy}

Sentinel node biopsy, which has been extensively validated in breast cancer and melanoma, is being used increasingly in the evaluation of penile cancer. The concept of the sentinel node, the first lymph node to contain metastatic cancer withn a tumor;s lymphatic basin, was introduced by Cabnas in $1977^{22}$. In an effort to more accurately stage and avoid extensive lymph node dissections in the majority of patients with penile cancer, Cabanas studied 100 cases of local T2 or greater disease using lymphangiograms, anatomic dissections, and / or microscopic evaluations. Anatomically, the sentine lymph node was discovered to be part of the lymphatic system around the superficial inferior epigastric vein, and theoretically, skip metastases beyond this node were supposedly rare. In the study, 46 sentinel node biopsies were performed, with 15 nodes being positive for metastatic disease and thus warranting a formal inguinal node dissection. no further involvement of other inguinal nodes was present in 12 of these 15 cases. Lymphatic channels draining into the iliac nodes without first draining into the sentinel lymph node were not seen, and inguinal-femoral lymph nodes were not involved with metastatic disease in the absence of sentinel lymph node positive disease. Based on these findings, Cabanas recommended bilateral sentinel node biopsy followed by inguino-femoral dissection only when biopsy of the sentinel node was positive. When the sentinel node was negative for metastatic disease, no further surgical treatment was recommended.

The reliability of this approach was limited by its relatively poor localization technique, and therefore it failed to gain widespread acceptance. Further refinements of the technique, including the use of radiolabeled colloids in conjunction with gamma imaging or gamma probeguided detection of the sentinel node at surgery, has resurrecteed the issue. By combining preoperative lymphatic mapping with intraoperative gamma probe detection, use of this nuclear medicine procedure to identify sentinel nodes is increasing.

Horenblas et al reported on a series of 55 patients with stage $\mathrm{T} 2$ or greater node-negative disease who underwent lymphoscintigraphy with technetium-99m nanocolloid injected intradermally around the tumor ${ }^{23}$. Sentinel nodes were found intraoperatively using patent blu dye injected intradermally around the tumor and a gamma detection probe. Regional node dissections were limited to patietns with tumor-positive sentinel nodes. A total of 108 sentinel nodes were removed, and 11 patients underwent a regional node dissection secondary to a sentinel node positive for metastatic disese. At a median follow-up of 22 months, 1 patient had nodal metastasis despite prior excision of tumor-free sentinel node. More recently, Valdes Olmos and colleagues reported on a series of 74 patients with T2 or greater disese clinically negative lymph nodes who underwent gamma mapping. The sentinel node visualization rate was $97 \%$, and lymphatic inuinal drainage was bilateral in $81 \%$ of cases. Bilateral lymph node drainage was asynchronous in $62 \%$, with half initially draining to the left inguinal nodes and half to the right. In total, 173 sentinel nodes were visualized, with 161 removed at surgery. Twenty two percent of patients had positive sentinel nodes and underwent a standard regional node dissection. Mean follow-up was 28 months. Two patients with negative sentinel nodes on initial evaluation developed nodal metastasis in the mapped lymphatic basin at follow-up. Both studies concluded that lymphoscintigraphy offers a valid, well tolerated method for lymphatic mapping and sentinel node identification. However, further technique can be accepted as standard practice.

\section{Chemotherapy for Penile Cancer}

The experience with cytotoxic drugs in penile and urethral carcinomas is limited to small series, with inconclusive results. Some response has been observed with cisplatin, bleomycin, and methotrexate, as well as with combinations of these agents. Shammas et all used cisplatin and 5 fluorouracil t treat 8 patients with advanced squamous cell carcinoma of the penis (Jackson stages III and IV $)^{24}$. Two patients achieved a partial response, 1 requiring a further operation and 1 requiring surgery with radiotherapy to achieve a complete response. These 2 patients were disese free at 32 and 57 months. Nonresponders had a survival range of 2 to 28 months after chemotherapy. Kattan and associates treated 14 patients with cisplatin based chemotherapy combined with other chemotherapeutic agents ${ }^{25}$. Thirteen patients were evaluable for response. Objective responses were achieved in 2 patients (15\%), with 1 complete and 1 partial. There were 2 patients with long term evidence of no disease among 12 patients with stage IV disease. These 2 patients received complementary irradiation in association with the chemotherapy. In this study, methotrexate based regimes seemed to be most effective. Pizzocaro et al evaluated neoadjuvant chemotherapy with combined vncristine, bleomycin, and methotrexate in 16 patients with fixed inguinal nodes ${ }^{26}$. Nine $(56 \%)$ of the 16 patients underwent successful 
surgical resection, and $5(31 \%)$ achieved 5 year disease free survival. The Southwest Oncology Group evaluated combination chemotherapy with cisplatin, bleomycin, and methotrexate in patients with metastatic disease. Of 40 patients evaluable for response, 5 complete responses and 8 partial responses occurred for a $32.5 \%$ response rate. The therapy was associated with excessive toxicity, with 5 treatment related deaths. Six of the remaining patients evaluable for toxicity had 1 or more life threatening toxic episodes.

\section{Conclusions}

Penile cancer, though a rare disease, poses many diagnostic, staging, and treatment challenges. Accurate treatment and staging of the primary lesion is important to aid in predicting the status of the regional lymph nodes. Modifying the groin lymph node dissection had lessened morbidity from this procedure, and sentinel node localization offers the possibility of less extensive surgery for some patients.

\section{Conflict of interest: None declared.}

\section{References:}

1. Maden C, Sherman KJ, Bechman AM et al: History of circumcision, medical conditions and sexual activity and risk of penile cancer. J NaH Cancer Inst 1993;85:19-24.

2. Schoen EJ, Oehrli M, Colly Cd, Machin G. The highly protective effect of newborn circumcision against invasive penile cancer. Paediatrics. 2000 Mar; 105 (3):E36.

3. Rubin MA, Kleter B, Zhou M, Ayala G, Cubilla AL, Quint WG, et al.: Detection and typing of human papillomavirus DNA in penile carcinoma: evidence for multiple independent pathways of penile carcinogenesis. Am J Pathol. 2001; 159: 1211-8.

4. Bezerra AL, Lopes A, Landmen G, Alencar GN, Torlori H, Villa LL. Clinicopathological features and human papilloma virus DNA prevalence of warty and squamous cell carcinoma of the penis. Am J Surg Pathol 2001; 25:673-678.

5. Dillner J, Meijer CJ, Horenblas S, von Krogh G. Etiology of squamous cell carcinoma of penis. Scand J Urol Nephrol Suppl 2000;205:189-193.

6. Jensen JB, Jensen KM, Ulhoi BP, et al. Sentinel lymph-node biopsy in patients with squamous cell carcinoma of the penis. BJU Int 2009; 103:11991203.
7. Cubilla AL, Reuter V, Velazquez E, Piris A, Saito $\mathrm{S}$, Young RH. Histologic classification of penile carcinoma and its relation to outcome in 61 patients with primary resection. Int J Surg Pathol 2001;9:111-120.

8. Jackson SM: The treatment of carcinoma of the penis. BrJ Surg. 1966;53:33.

9. American Joint committee on cancer: TNM classification Genitourinary sites. 1996.

10. Leijte JA, Gallee M, Antonini N, Horenblas S. Evaluation of current TNM classification of penile carcinoma. J Urol 2008; 180:933-938. According to this study, the current TNM classification (unchanged since 1987) is not able to accurately identify significant differences in prognosis among some $\mathrm{T}$ and $\mathrm{N}$ stages. The authors propose a new TNM classification for penile cancer.

11. Horenblas $\mathrm{S}$, van Tinteren $\mathrm{H}$. Squamous cell carcinoma of the penis. IV. Prognostic factors of survival: analysis of tumor, nodes and metastasis classification system. J Urol 1994; 151:1239-1243.

12. 10 Zhu $Y$, Ye DW, Yao XD, et al. The value of squamous cell carcinoma antigen in the prognostic evaluation, treatment monitoring and followup of patients with penile cancer. J Urol 2008; 180:20192023.

13. Bissada NK, Yakout HH, Fahmy WE, Gayed MS, Touijer AK, Greenc GF, Hanash KA, Multiinstitutional long-term experience with conservative surgery for invasive penile carcinoma. J Urol 2003; 169:500-502.

14. Mohs FE, Snow SN, Lorson PO. Mohs micrographic surgery for penile tumor, Urol Clin North Am 1992;19:291-304.

15. Hofstetter A, Staehler G, Keiditsch E, Frank F, Local irradiation by laser of a penile carcinoma. Fortschr Med 1978; 96:369-371.

16. Agraval A, Paio, Ananthakrishmn N, Smile SR, Ratnakar C. The histological extent of the local spread of carcinoma of the penis and its therapeutic implications. BJU int 2000;85:299-301.

17. Minhas $\mathrm{S}$, Kayes $\mathrm{O}$, Hegarty $\mathrm{P}$, et al. What surgical resection margins are required to achieve oncological control in men with primary penile cancer? BJU Int 2005; 96:1040-1043. 
18. Davis JW, Schellhammer PF, Schlossberg SM. Conservative surgical therapy for penile and urethral carcinoma. Urology 1999; 53:386-392.

19. Catalona WJ: Modified inguinal lymphadenectomy for carcinoma of the penis with preservation of sopherous veins: Technique and preliminary results. J Urol 1988; 29:608-610.

20. Colberg JW,Andriole GL, Catalona WJ. Long-term follow-up of men undergoing modified inguinal lymphadenectomy for carcinoma of the penis. $\mathrm{Br}$ J Urol. 1997;79:54-57.

21. Solsona E, Iborra I, Rubio J, Casanova JL, Ricos $\mathrm{JV}$, Calabuig C. Prospective validation of the association of local tumor stage grade as a predictive inguinal lymph nodes. J Urol 2001; 165:1506-1509.

22. Cabanos RM, An approach for the treatment of penile carcinoma. Cancer 1977;39:456-466.
23. Horenblas S. Lymphadenectomy for squamous cell carcinoma of the penis. Part-2; the role and technique of lymph node dissection. BJU Int 2001;188:473-483.

24. Shammas F.V., Ous S., Fossa S.D. (1992) Cisplatin and 5-fluorouracil in advanced cancer of the penis. J Urol 147: 630-632. [PubMed]

25. Kattan J., Culine S., Droz J.P., Fadel E., Court B., Perrin J.L., et al. (1993) Penile cancer chemotherapy: twelve years' experience at Institut Gustave-Roussy. Urology 42: 559-562. [PubMed]

26. Pizzocaro G, Algaba F, Horenblas S, et al. EAU Guidelines on penile cancer. http://www.uroweb.org/ fileadmin/tx_eauguidelines/2009/Full/ Penile_Cancer.pdf; 2009. A complete update addressing many aspects of penile cancer management. Proposal for new TNM classification is available, as well as recommendations for treatments and follow-up. 